\title{
A Push-Enabling Scheme for Live Streaming System in Content-Centric Networking
}

\author{
Kwangsoo Kim, Seungoh Choi, Seongmin Kim, and Byeong-hee Roh \\ Dept. of Computer Engineering in Grad. School at Ajou University, South Korea
}

\begin{abstract}
Content-Centric Networking $(\mathrm{CCN})$ is a promising candidate for Future Internet architecture. This pulling-based networking scheme has a weakness about live streaming due to their request method, which means every segment of contents should be requested by Interest, respectively and consequently. We propose an enabling scheme for push mechanism to $\mathrm{CCN}$ to overcome the ineffectiveness. Push Interest, which is not removed after delivering the segment, needs to be added to the network design. Main contribution of this paper is suggestion of the signaling process for pushing. We add two types of packet and some rules on the $\mathrm{CCN}$, which are co-operable with original pulling protocol. We expect the number of interest will be dramatically decreased in live streaming.
\end{abstract}

\section{Categories and Subject Descriptors}

C.2.1 [Computer-Communication Networks]: Network Architecture and Design-Store and forward networks

\section{Keywords}

Content Centric Networking; Live Streaming; Pushing; Interest Packet

\section{INTRODUCTION}

The Content-Centric Networking (CCN) is one of the candidates to design the Future Internet architecture as a clean-slate according to the era of tremendous amount of exchanging contents. $\mathrm{CCN}$ has very strong advantages for on-demand service due to in-network cache. However, the user should request the contents to server because the new content segments are created on server in case of live streaming. In addition, the content should exist to retrieve by the requester, or the Interest will be pended without replying any data.

Thus, it is necessary to make an effort to overcome these disadvantages in live streaming system. We propose a push enabling scheme in $\mathrm{CCN}$, which is equivalent to the multicast scheme in IP forwarding network, by introducing Push Interest and Push Data packets. Our push enabling protocol can work on the basic rules of $\mathrm{CCN}$ without breaking rules. We expect our scheme works well with constant number of Interest packets for streaming live contents.

Permission to make digital or hard copies of all or part of this work for personal or classroom use is granted without fee provided that copies are not made or distributed for profit or commercial advantage and that copies bear this notice and the full citation on the first page. Copyrights for components of this work owned by others than ACM must be honored. Abstracting with credit is permitted. To copy otherwise, or republish, to post on servers or to redistribute to lists, requires prior specific permission and/or a fee. Request permissions from Permissions@acm.org.

CoNEXT Student Workhop'13, December 09 2013, Santa Barbara, CA, USA Copyright 2013 ACM 978-1-4503-2575-2/13/12 ...\$15.00.

http://dx.doi.org/10.1145/2537148.2537162

\section{RELATED WORKS}

2.1 Content-Centric Networking [1]

The basic CCN protocol operates request and reply mechanism called pulling, that the user can obtain only the content he requested. There are two features in $\mathrm{CCN}$ (i.e. in-network caching and Interest aggregation) that dramatically reduce the amount of traffic on the entire network.

Many kinds of Internet services are provided using Push scheme currently, which sends data to the subscribers without any request of further specific segments. In $\mathrm{CCN}$, however, if there was no Interest in Pending Interest Table (PIT), the forwarded Data should be discarded because it is regarded as an unsolicited chunk. It means the push services currently served are fundamentally blocked in $\mathrm{CCN}$.

To obtain large amount of segments of content consecutively, users can use "pipeline" scheme suggested in VoCCN [2]. It requests further segments before receiving previous segments. This scheme can reduce the inter-arrival time dramatically rather than lock-step scheme. However it still needs the same amount of Interest packets to the requesting segment even though the user requests consecutive segments. With this reason, it is needed an efficient scheme to transfer certain content types such as large file and live contents in $\mathrm{CCN}$.

2.2 Persistent Interest [3] (Long term Interest [4]) Another trial to serve the push scheme is Persistent Interest (PI) scheme in [3] and its implementation is [4]. PI makes a push tree for certain content, and it is not removed after matched data was forwarded. It can aggregate other segments by channel name, but it only considers Interest packet. Even though the normal Interest for pulling and the PI for pushing exist as mixed, normal data and pushed data are not identified in the router. Thus, the two types of data will get mixed normal data is flowed through unintentional push tree. Moreover, anyone can join existing push tree if the user knows the channel name without authority. Therefore, the push scheme needs to be able to overcome these problems.

\section{PUSH ENABLED CCN}

\subsection{Requirements}

We agree that it is the simplest way to make a push path in $\mathrm{CCN}$ that the Interest should not be removed when the matched Data is received as mentioned in previous section. User should request each segment respectively when requesting large content divided into several segments. There are some requirements for transferring live contents more efficiently. Firstly, network should support the push scheme that forwards data to the destination without requests. In $\mathrm{CCN}$, transferring data without any request is impossible. Thus, we introduce Push Request as a trigger and Push Interest as an indicator of confirmed path. Secondly, every push path should be securely set not to use the path maliciously by attackers (a.k.a. DDoS) even though our push scheme, which is 
similar to the IP push scheme exposed to DDoS in general, is enabled to $\mathrm{CCN}$.

Table 1. The Usage of Push Enabling Packets (added features)

\begin{tabular}{|c|c|l|}
\hline $\begin{array}{c}\text { Message } \\
\text { Type }\end{array}$ & $\begin{array}{c}\text { Packet } \\
\text { Type }\end{array}$ & \multicolumn{1}{|c|}{ CCN Name } \\
\hline Push Request & Interest & PushReq/domain/filename.ext/ID \\
\hline Push Confirm & Data & PushReq/domain/filename.ext/ID \\
\hline Push $^{2}$ & Interest & Push/domain/filename.ext \\
\hline Push $^{3}$ & Data & Push/domain/filename.ext/Syy \\
\hline
\end{tabular}

\subsection{The Push-Enabling Protocol}

The usage of additional Interest/Data packets as push-enabler is shown in Table 1. We add prefixes which have different actions from original types. Each type of packets can behave well without breaking the existing rules of CCN. The PushReq and Push keyword are set as reserved prefixes. The version information of the content object is omitted in this paper because we focus several consecutive segments of content object rather than several version of content.

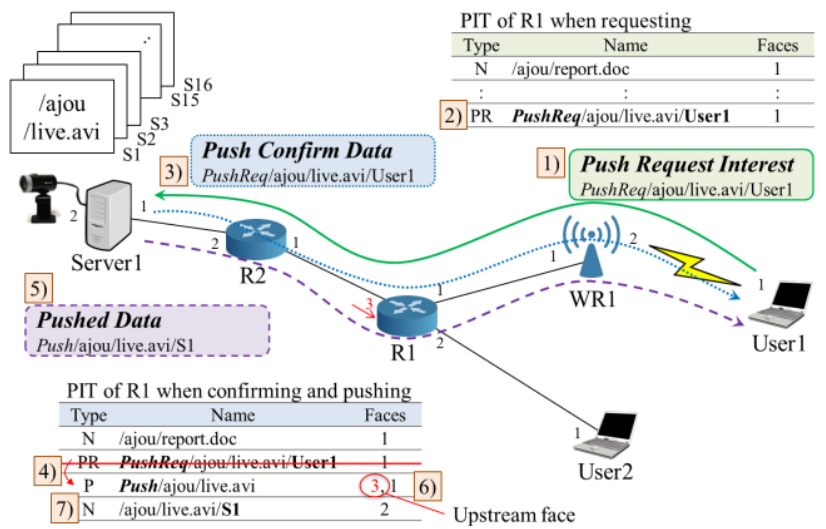

Figure 1. The push association using push enabler packets

The overall protocol is described in Figure 1. The solid, dotted, and dashed arrows stand for Push Request, Push Confirm, and Push Data, respectively. When User1 wants to get a live content using our push scheme, 1) User1 sends a Push Request message with modified name including PushReq keyword and his any unique ID to Server1. 2) Each router stores the received PushReq Interest packet to PIT, and forwards it to server. (This push association is one-to-one between server and user.) 3) After receiving PushReq Interest, the server sends PushReq Data packet as a Push Confirm if user's request is allowed. 4) This Push Confirm Data packet, whose prefix is PushReq, is forwarded toward the user without caching on router and it converts the PushReq Interest stored in PIT to Push Interest, which constructs

${ }^{1}$ ID is whatever unique identifier of node, thus the push request can be identified.

${ }^{2}$ Push Interest is not an actual exchanging packet by nodes, but changed from Interest of Push Request in PIT to aggregate the all content segments.

${ }^{3}$ However, Push Data is a real Data packet to specify the packet from server, and it is also cached in CS without Push keyword after router forwards the Data packet toward the push path. It is exactly same as original Data packet without Push keyword. confirmed path. 5) Every contents newly created at the server can be pushed without additional Interest because the push path was already enabled. Server1 sends the pushing Data with Push prefix which represents it is pushed by server, and each routers can distinguish to traditional on-demand Data and pushed Data. Pushed Data finds the outgoing faces from Push Interest in PIT, and then it is forwarded to the outgoing faces.

Additionally, 6) The Push Interests in PIT contain an upstream face to the server to specify the designated stream. Thus, any other nodes cannot misuse the path. The router can forward the push data from only the upstream face, which is the first item of the face list, to other faces. (E.g. the red-colored number 3 of Push Interest at Step 6 in Figure 1 represents the upstream face.) 7) Conventional on-demand Interest can request without modification. Although there are one or more Interests having the same contents as Push Interest, they can coexist because normal Interest will be processed after pushing.

\subsection{Security Considerations}

Every push path should be set by only the server and unsolicited Push Confirm should not be allowed to prevent DDoS attack caused by numerous push confirms from malicious nodes. Thus, in the Step 3 and 4 the Push Confirm packet (i.e. PushReq Data) should be established at the server and the Push Confirm should convert only matched Push Request to Push Interest at the router. These restrictions can prevent to flood traffic by creating an arbitrary push path from malicious node. Setting the upstream face can block to misuse the existing path like flooding.

\section{DISCUSSION AND FUTURE WORKS}

We proposed a concept of push enabling scheme in CCN. As giving a specific behavior to the packets to enable push, we expect that it can achieve more efficient bandwidth usage than basic protocol of CCN. Our scheme not only can provide the complete multicast scheme equivalent to that in IP but also does contribute to encourage the QoS and user's satisfaction, especially, in the services transfer large amount of consecutive segments such as large file transmission and live streaming services.

Further works will be followed. Firstly, our scheme will be proved by testing using packet level CCN simulator we implemented using OPNET Modeler w.r.t. network load, memory consumption, and processing time of new packets. Secondly, robustness against the DDoS attack also needs to be proved. Lastly, the maintenance scheme for push tree needs to be added.

\section{ACKNOWLEDGMENT}

This research was supported by Basic Science Research Program through the NRF of Korea funded by the Ministry of Education (NRF-2011-0025544)

\section{REFERENCES}

[1] Jacobson, Van, Diana K. Smetters, James D. Thornton, Michael F. Plass, Nicholas H. Briggs, and Rebecca L. Braynard. 2009. Networking named content. In Proceedings of the 5th international conference on Emerging networking experiments and technologies (CoNEXT '09). ACM, New York, NY, USA, 1-12. DOI=10.1145/1658939.1658941 http://doi.acm.org/10.1145/1658939.1658941

[2] Jacobson, Van, Diana K. Smetters, Nicholas H. Briggs, Michael F. Plass, Paul Stewart, James D. Thornton, and 
Rebecca L. Braynard. 2009. VoCCN: voice-over contentcentric networks. In Proceedings of the 2009 workshop on Re-architecting the internet (ReArch '09). ACM, New York, NY, USA, 1-6. DOI=10.1145/1658978.1658980 http://doi.acm.org/10.1145/1658978.1658980

[3] Tsilopoulos, Christos and George Xylomenos. 2011. Supporting diverse traffic types in information centric networks. In Proceedings of the ACM SIGCOMM workshop on Information-centric networking (ICN '11). ACM, New York, NY, USA, 13-18. DOI=10.1145/2018584.2018588 http://doi.acm.org/10.1145/2018584.2018588

[4] Yao, Chunfeng, Lingyuan Fan, Zhefeng Yan, and Yanping Xiang. 2012. Long-Term Interest for Realtime Applications in the Named Data Network. In Proceedings of the AsiaFI 2012 Summer School (AsiaFI '12). ACM, Kyoto, Japan. 